\title{
PRIME IDEALS IN POLYNOMIAL RINGS OVER ONE-DIMENSIONAL DOMAINS
}

\author{
WILLIAM HEINZER AND SYLVIA WIEGAND
}

\begin{abstract}
Let $R$ be a one-dimensional integral domain with only finitely many maximal ideals and let $x$ be an indeterminate over $R$. We study the prime spectrum of the polynomial ring $R[x]$ as a partially ordered set. In the case where $R$ is countable we classify $\operatorname{Spec}(R[x])$ in terms of splitting properties of the maximal ideals $\mathrm{m}$ of $R$ and the valuative dimension of $R_{\mathrm{m}}$.
\end{abstract}

Let $R$ be as in the abstract. Since $\operatorname{Spec}(R)$ is finite, $\operatorname{Spec}(R[x])$ is Noetherian; Ohm and Pendleton show that every finitely generated algebra over a ring with Noetherian spectrum again has Noetherian spectrum [OP, Corollary 2.6, page 634]. Thus in our setting, the partial order on $\operatorname{Spec}(R[x])$ uniquely determines $\operatorname{Spec}(R[x])$ as a topological space with the Zariski topology.

In the case where $R$ is a countable one-dimensional local Noetherian domain, we show in [HW, Theorem 2.7] that there are precisely two possibilities for $\operatorname{Spec}(R[x])$, one of which occurs when $R$ is Henselian and the other when $R$ is not Henselian. We also show that if $R$ is a countable one-dimensional semilocal Noetherian domain having more than one maximal ideal, then the spectrum of $R[x]$ is uniquely determined up to isomorphism by the number of maximal ideals of $R$. (In this latter case, $R$ cannot be Henselian.)

An important concept related to our work here and in [HW] is the $n$-split property introduced by McAdam in [Mc] for a prime ideal $P$ of an integral domain $D$ : If $D^{a}$ is the integral closure of $D$ in an algebraic closure of the quotient field of $D$, then $P$ is said to be $n$-split if there are exactly $n$ primes in $D^{a}$ which lie over $P$. (Possibly $n=\infty$.) We show in [HW, Theorem 1.1] that every prime ideal of a Noetherian domain $D$ is either 1-split or $\infty$-split and if $P$ is a nonzero prime ideal of $D$ that is 1 -split, then $D$ is local with maximal ideal $P$.

It is noted in [HW, Example 1.6] that for every positive integer $n$, there exists a one-dimensional non-Noetherian local integral domain with $n$-split maximal ideal. Using the more varied behavior of the splitting of prime ideals in a non-Noetherian domain, we show in this article that the possible spectra of the polynomial ring $R[x]$ over a one-dimensional non-Noetherian local domain $R$ are considerably more varied than in the Noetherian case. Theorem 3.1 demonstrates that the $n$-split property in $R$ yields a distinguishing characteristic of $\operatorname{Spec}(R[x])$.

Received by the editors July 12, 1993; originally communicated to the Proceedings of the AMS by Wolmer V. Vasconcelos.

1991 Mathematics Subject Classification. Primary 13E05, 13F20, 13G05, 13H99, 13 J15. 
Another factor which contributes to the greater variety in spectra for $R[x]$ in the non-Noetherian case is variation in the Krull dimension of $R[x]$. In general, if $R$ is an integral domain of dimension one, $\operatorname{dim}(R[x])$ is either two or three [S, Theorem 2, page 506], and $\operatorname{dim}(R[x])=3$ if and only if $R$ has valuative dimension greater than one [G, (30.12) and (30.14), pages 363-364]. If $R$ is a countable one-dimensional local domain, we show the existence of infinitely many two-dimensional possibilities for $\operatorname{Spec}(R[x])$ and also infinitely many three-dimensional possibilities (Theorems 3.3 and 3.6). We observe in Theorem 2.1, however, that the $j$-spectra of all these rings are isomorphic. (The $j$-spectrum of $R[x], j-\operatorname{Spec}(R[x])$, is the partially ordered set of $j$-primes of $R[x]$-those prime ideals of $R[x]$ which are intersections of maximal ideals. )

All rings we consider are assumed to be commutative with identity. In general our notation is as in Matsumura [M]. In particular, "local" and "semilocal" rings are not necessarily Noetherian.

\section{INTRODUCTION AND BACKGROUND}

We will be using the following conventions, notation, definitions, and theorems from [Mc], [HLW1], [HLW2] and [HW]:

1.1 Notation. For $U$ a partially ordered set of finite dimension, elements $u, v$ of $U$, and $T$ a finite subset of $U$, we set

$$
\begin{aligned}
\mathrm{G}(u) & =\{w \in U \mid w>u\}, \\
\mathrm{L}_{\mathrm{e}}(T) & =\{w \in U \mid \mathrm{G}(w)=T\}, \\
\mathscr{M}(U) & =\{\text { maximal elements of } U \text { of maximal height }\} .
\end{aligned}
$$

(The notation stands for the "greater" set, the "exactly-less-than" set, and the "maximal" set.)

1.2 Definition. For $P$ a prime ideal of an integral domain $D$ and $D^{a}$ the integral closure of $D$ in an algebraic closure of the quotient field of $D, P$ is said to be $n$-split if there are exactly $n$ primes in $D^{a}$ (possibly $n=\infty$ ) which lie over $P$. A local domain $(D, \mathbf{m})$ is said to be $n$-split if $\mathbf{m}$ is $n$-split.

1.3 Theorem [HW, Theorem 2.7]. Suppose $R$ is a (countable) Noetherian onedimensional domain with exactly $m$ maximal ideals. Let $U=\operatorname{Spec}(R[x])$, where $x$ is an indeterminate over $R$. Then:

(1) $U$ has the following properties:

(P0) $U$ is countable (if $R$ is countable).

(P1) $U$ has a unique minimal element $u_{0}$.

(P2) $U$ has dimension two.

(P3) $U$ has infinitely many height-one maximal elements.

(P4) $U$ has exactly $m$ height-one nonmaximal j-elements. We denote these elements $u_{1}, u_{2}, \ldots, u_{m}$. They satisfy:

(i) $\mathrm{G}\left(u_{1}\right) \cup \cdots \cup \mathrm{G}\left(u_{m}\right)=\mathscr{M}(U)$,

(ii) $\mathrm{G}\left(u_{i}\right) \cap \mathrm{G}\left(u_{j}\right)=\varnothing$ for $i \neq j$, and

(iii) $\mathrm{G}\left(u_{i}\right)$ is infinite for each $i, 1 \leq i \leq m$.

(P5) For each height-one element $u \neq u_{i}, \mathrm{G}(u)$ is finite.

(2) If $m=1$ and $R$ is 1-split, $U$ satisfies

(P6 1$)$ For each finite subset $T$ of $\mathscr{M}(U)$ of cardinality greater than one, $\mathrm{L}_{\mathrm{e}}(T)$ is empty. For each element $t$ of $\mathscr{M}(U), \mathrm{L}_{\mathrm{e}}(\{t\})$ is infinite. 
(3) Otherwise, that is, if every maximal ideal of $R$ is $\infty$-split, then $\operatorname{Spec}(R[x])$ satisfies

$\left(\mathrm{P}_{\infty}\right)$ For each nonempty finite subset $T$ of $\mathscr{M}(U), \mathrm{L}_{\mathrm{e}}(T)$ is infinite.

(4) If $V$ is a countable partially ordered set satisfying properties (P0)-(P5) of Part (1) and $\left(\mathrm{P} 6_{1}\right)$ or $\left(\mathrm{P} 6_{\infty}\right)$ (whichever holds for $\left.U\right)$, then $V$ is orderisomorphic to $U$.

In [HW], the ring $R=S^{-1} \mathbf{Z}$, where $S=\mathbf{Z}-\bigcup_{i=1}^{m} \mathbf{p}_{i}$ and $\left\{\mathbf{p}_{i} \mid 1 \leq i \leq m\right\}$ is a finite set of primes, is given as an example where $\operatorname{Spec}(R[x])$ is of $\left(\mathrm{P}_{\infty}\right)$ type.

Pictorially, the $\left(\mathrm{P} 6_{\infty}\right)$ type looks like the diagram in Figure 1.

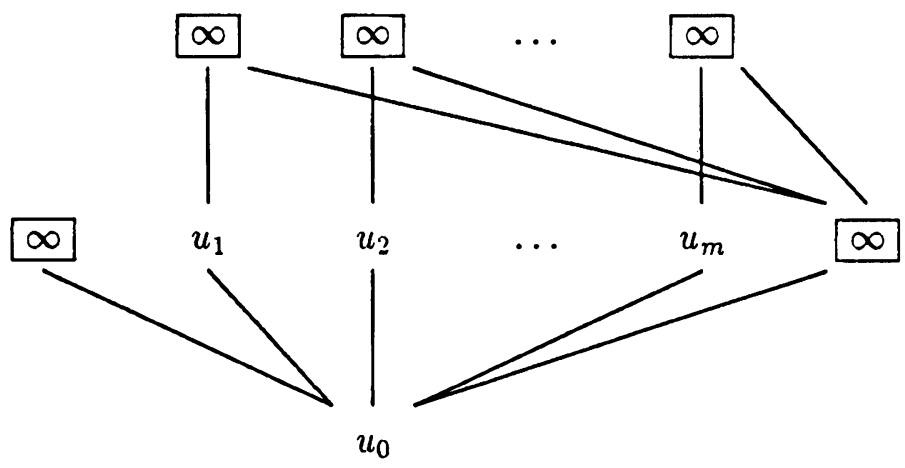

FIGURE 1

The relationships of the lower-right boxed section, determined by (P5) and $\left(\mathrm{P} 6_{\infty}\right)$, are too complicated to display.)

1.4 Definition. The valuative dimension of an integral domain $D$ is the supremum of the dimension of valuation domains between $D$ and the fraction field of $D$.

The valuative dimension of $D$ is related to the dimension of the polynomial ring $D[x]$ (cf. $[\mathrm{J}]$ and $[\mathrm{G}$, Theorem 30.9, page 360]).

Here are two examples, illustrating the dimension two and dimension three possibilities for $\operatorname{Spec}(R[x])$ :

1.5 Example. Let $R=k+z k(y)[z]_{(z)}$, where $k$ is a field and $y$ and $z$ are indeterminates over $k$. Then $R$ is one-dimensional, but the polynomial ring $R[x]$ is three-dimensional. A simple way to see this is to observe that the $R$-homomorphism of $R[x]$ to $R[y]$ taking $x$ to $y$ is onto, $R[y]$ is twodimensional, and the kernel of this homomorphism is a height-one prime ideal of $R[x]$. In this example the valuative dimension of $R$ is two.

1.6 Example. Let $R=k\left[\left\{y^{r>0} \mid r \text { is positive rational }\right\}\right]_{\mathrm{m}}$, where $k$ is a field, $y$ is an indeterminate, and $\mathrm{m}$ is the maximal ideal generated by the $y^{r}$. In this example, $R$ is a non-Noetherian rank-one valuation domain and $\operatorname{Spec}(R[x])$ has dimension two. 


\section{THE $j$-SPECTRUM OF THE POLYNOMIAL RING $R[x]$}

For a one-dimensional non-Noetherian domain $R$ with finite spectrum, we observe that the $j$-spectrum of $R[x]$ is the same as in the Noetherian case [HLW1, Theorem 1.2].

2.1 Theorem. If $R$ is a one-dimensional (countable) domain with exactly $m$ maximal ideals, then $U=j-\operatorname{Spec}(R[x])$ has the following properties:

( $\mathrm{P} 0) \quad U$ is countable (if $R$ is countable).

(P1) $U$ has a unique minimal element $u_{0}$.

(P2) $U$ has dimension 2.

(P3) $U$ has infinitely many height-one maximal elements.

$\left(\mathrm{P}_{j}\right) U$ has exactly $m$ height-one nonmaximal elements. We denote these elements $u_{1}, u_{2}, \ldots, u_{m}$. They satisfy:

(i) $\mathrm{G}\left(u_{1}\right) \cup \cdots \cup \mathrm{G}\left(u_{m}\right)=\mathscr{M}(U)$,

(ii) $\mathrm{G}\left(u_{i}\right) \cap \mathrm{G}\left(u_{j}\right)=\varnothing$ for $i \neq j$, and

(iii) $\mathrm{G}\left(u_{i}\right)$ is infinite for each $i, 1 \leq i \leq m$.

Proof. Since $\operatorname{Spec}(R[x])$ is Noetherian, each prime in $R[x]$ is the radical of a finitely generated ideal, so (P0) holds. For property (P3), we make use of the fraction field $K$ of $R$. Since $K[x]$ is a localization of $R[x]$, the height-one primes of the principal ideal domain $K[x]$ contract to height-one primes of $R[x]$. If $a$ is any nonzero element in the intersection of the maximal ideals of $R$, then we have $(a x-1) R[x]=(a x-1) K[x] \cap R[x]=P_{a}$, a height-one prime ideal of $R[x]$. Moreover, $R[x] / P_{a}=R[1 / a]=K$, a field. Therefore each $P_{a}$ is a height-one maximal ideal of $R[x]$. Since the intersection of the finitely many maximal ideals of $R$ is an infinite set, there are infinitely many distinct height-one maximal ideals $P_{a}$ of $R[x]$.

We have $(0)=\bigcap\left\{P_{a} \mid a \in \bigcap_{i=1}^{m}\left\{\mathbf{m}_{i}\right\}\right\}$, so (0) is a $j$-prime of $\operatorname{Spec}(R[x])$ and (P1) holds, since every $j$-prime is comparable with $(0)$.

For (P2) and $\left(\mathrm{P}_{j}\right)$, observe that for each maximal ideal $\mathbf{m}_{i}$ of $R$ we have $R[x] / \mathbf{m}_{i}[x] \cong\left(R / \mathbf{m}_{i}\right)[x]$, a polynomial ring in one variable over a field. Hence $\mathbf{m}_{i}[x]$ is a $j$-prime of $R[x]$ of dimension one. Moreover, if $P$ is a prime ideal of $R[x]$ of height at least two, then $P \cap R$ is nonzero and hence $\mathbf{m}_{i}[x] \subseteq P$ for some maximal ideal $\mathbf{m}_{i}$ of $R$. Thus to complete the proof of Theorem 2.1, setting $u_{i}=\mathbf{m}_{i}[x]$, for $1 \leq i \leq m$, it suffices to observe that $\mathbf{m}_{i}[x]$ does not properly contain a nonzero $j$-prime of $R[x] .^{1}$ If $Q$ is a nonzero nonmaximal $j$-prime of $R[x]$, then $Q$ is an intersection of maximal ideals of $R[x]$ of height greater than one. Each of these maximal ideals has a nonzero intersection with $R$. It follows that $Q \cap R$ contains the intersection of all the (finitely many) nonzero prime ideals of $R$, and hence $Q \cap R=\mathbf{m}_{i}$, for some $i$, which implies that $\mathbf{m}_{i}[x]=Q$. Therefore $u_{i}=\mathbf{m}_{i}[x], i=1, \ldots, m$, are precisely the nonzero nonmaximal $j$-primes of $R[x]$. Thus (P2) and $\left(\mathrm{P}_{j}\right)$ hold.

2.2 Remarks. (1) The properties $(\mathrm{P} 0)-\left(\mathrm{P} 4_{j}\right)$ characterize a partially ordered set $U$ having these properties in the sense that every partially ordered set with these properties is order-isomorphic to $U$.

(2) If $R$ is uncountable, then (P1)-(P4 $\left.{ }_{j}\right)$ hold, but, obviously, different cardinality assertions are necessary to obtain a characterization of $j$-Spec $(R[x])$.

${ }^{1}$ If $R_{\mathbf{m}_{i}}$ has valuative dimension greater than one, then $\mathbf{m}_{i}[x]$ has height two in $R[x]$. 
The referee has observed that in several of our results, such as Theorem 2.1, the countable hypothesis on $R$ could be replaced with the hypothesis that $R$ has cardinality $\alpha$ to thereby deduce a sharper ^ esult with the word 'infinite' replaced by 'of cardinality $\alpha$ '.

(3) If $D$ is a two-dimensional domain such that each element of the $j$ spectrum has the same height in the $j$-spectrum of $D$ as in the entire spectrum of $D$, then $\operatorname{Spec}(D)$ differs from $j$ - $\operatorname{Spec}(D)$ solely by the additional height-one elements which are not $j$-primes. On the other hand, if some elements of the $j$-spectrum do not have the same height in the $j$-spectrum of $D$ as in the entire spectrum of $D$, then $\operatorname{Spec}(D)$ is different from $j-\operatorname{Spec}(D)$ in other ways. This is illustrated in Theorem 3.6.

\section{THE SPECTRUM OF $R[x]$}

Theorem 3.1 shows the effect on $\operatorname{Spec}(R[x])$ of the $n$-split property of $R$.

3.1 Theorem. Let $n$ be either a positive integer or $\infty$ and assume that $(R, \mathbf{m})$ is an $n$-split one-dimensional local domain. Then $U=\operatorname{Spec}(R[x])$ satisfies:

$\left(\mathrm{P}_{n}\right)$ For each finite subset $T$ of $\mathscr{M}(U)$ :

(i) if $|T| \leq n$, then $\left|\mathrm{L}_{\mathrm{e}}(T)\right|=\infty$, and

(ii) if $|T|>n$, then $\mathrm{L}_{\mathrm{e}}(T)=\varnothing$.

Proof. Let $K^{a}$ be an algebraic closure of $K$, the quotient field of $R$, and let $R^{a}$ be the integral closure of $R$ in $K^{a}$. By the definition of $n$-split, $R^{a}$ has exactly $n$ maximal ideals, which we call $\mathscr{N}_{1}, \ldots, \mathscr{N}_{n}$.

Suppose $n<\infty$ and $|T|>n$. We show that every nonmaximal height-one prime $Q$ of $R[x]$ such that $Q \nsubseteq \mathrm{m}[x]$ is contained in at most $n$ maximal ideals of $R[x]$. (If $n=\infty$, this is always true.) It will follow that $\mathrm{L}_{\mathrm{e}}(T)=\varnothing$.

Now $Q$ has the form $(f(x)) K[x] \cap R[x]$, where $f(x) \in R[x]$ is irreducible over $K[x]$. Thus $R[x] / Q=R[\theta]$, where $\theta$ is a root of $f(x)$, so $\theta$ is algebraic over $R$. It suffices to show that $R[\theta]$ has at most $n$ maximal ideals. We prove this via two claims:

Claim 1. Let $\left(S_{i}, \mathscr{M}_{i}\right)$ denote $R^{a}$ localized at $\mathscr{N}_{i}$, for $1 \leq i \leq n$. Then $S_{i}[\theta]=S_{i}$ or $S_{i}[\theta]=K^{a}$.

Proof of Claim 1. Since $S_{i}$ is integrally closed and $\theta \in K^{a}$, the quotient field of $S_{i}$, the kernel of the canonical $S_{i}$-homomorphism $S_{i}[x] \rightarrow S_{i}[\theta]$ is generated by elements of the form $b x-a$, where $b, a \in S_{i}$ [N, Theorem 11.13]. Now there are three cases to consider:

Case i: Suppose that $\theta$ is a root of $b x-a$, where $b \notin \mathscr{M}_{i}$. In this case, $\theta \in S_{i}$, and so $S_{i}[\theta]=S_{i}$.

Case ii: Suppose that $\theta$ is a root of $b x-a$, where $b \in \mathscr{M}_{i}, a \notin \mathscr{M}_{i}$. Then $S_{i}[\theta]=S_{i}[1 / b]$. Since $S_{i}$ is one-dimensional local with $b$ in its maximal ideal, we have $S_{i}[\theta]=K^{a}$.

Case iii: Suppose that every $b x-a$ in the kernel of the $S_{i}$-homomorphism $S_{i}[x] \rightarrow S_{i}[\theta]$ has $b \in \mathscr{M}_{i}, a \in \mathscr{M}_{i}$. Then $\operatorname{kernel}\left(S_{i}[x] \rightarrow S_{i}[\theta]\right) \subseteq \mathscr{M}_{i}[x]$, which implies that $Q=\operatorname{kernel}(R[x] \rightarrow R[\theta]) \subseteq \mathscr{M}_{i}[x] \cap R[x]=\mathbf{m}[x]$, a contradiction to the choice of $Q$. Thus there is no case iii, and so Claim 1 is proved.

Claim 2. $R^{a}[\theta]$ has at most $n$ maximal ideals. 
Proof of Claim 2. Let $\mathscr{N}$ be a maximal ideal of $R^{a}[\theta]$. Then $\mathscr{N} \cap R^{a}=\mathscr{N}_{i}$, for some $i$ with $1 \leq i \leq n$. We have $S_{i}[\theta] \subseteq\left(R^{a}[\theta]\right)_{\mathscr{N}}$. If $S_{i}[\theta]=K^{a}$, then $\left(R^{a}[\theta]\right)_{\mathcal{N}}=K^{a}$, which is impossible since $\mathscr{N}$ is a nonzero prime ideal of $R^{a}[\theta]$. Thus $\theta \in S_{i}$, and so $R^{a}[\theta] \subseteq S_{i}$. Hence $\left(R^{a}[\theta]\right)_{\mathcal{N}}=S_{i}$. This holds for all $\mathscr{N}$ with $\mathscr{N} \cap R^{a}=\mathscr{N}_{i}$. Thus there can be at most one maximal ideal of $R^{a}[\theta]$ lying over each maximal ideal of $R^{a}$; so $R^{a}[\theta]$ has at most $n$ maximal ideals.

By the Lying Over Theorem it follows that $R[\theta]$ has at most $n$ maximal ideals, and so $R[x]$ has at most $n$ maximal ideals containing $Q$.

Our proof of $\left(\mathrm{P}_{n}(\mathrm{i})\right)$ is similar to the proof of Theorem 2.7 of [HW] and goes back to McAdam in [Mc]. Let $t$ be a positive integer with $t \leq n$ and let $T$ be a set of $t$ maximal ideals of $R[x]$ of height at least two. Then $T=\left\{\left(\mathbf{m}, g_{i}(x)\right)\right\}_{i=1}^{t}$, where the $g_{i}(x)$ are monic and are irreducible mod $\mathbf{m}$. (The maximal ideals of $R[x]$ are of this form, because $(R / \mathbf{m})[x]$ is a principal ideal domain.) Since $\mathbf{m}$ is $n$-split, $R^{a}$ has $n$ distinct primes lying over $\mathbf{m}$; also each $g_{i}(x)$ splits into linear factors over $K^{a}$. Thus there exists a field $L$, $K \subseteq L \subseteq K^{a}$, such that $[L: K]<\infty$, each $g_{i}(x)$ splits into linear factors over $L$, and the integral closure $R^{\prime}$ of $R$ in $L$ has at least $t$ distinct prime ideals. Since $g_{i}(x)$ is monic and $R^{\prime}$ is integrally closed, $g_{i}(x)$ splits into linear factors in $R^{\prime}[x]$. Let $b_{i} \in R^{\prime}$ be a root of $g_{i}(x)$. By adjoining to $R$ an appropriate finite number of elements of $R^{\prime}$ we can obtain a ring $R^{*}$ such that $b_{1}, \ldots, b_{t} \in R^{*}, R^{*}$ has at least $t$ distinct maximal ideals, $R \subseteq R^{*} \subseteq$ $R^{\prime}$, and $R^{*}$ is a finitely generated $R$-module. Let $\mathbf{m}_{1}, \ldots, \mathbf{m}_{t}$ be distinct maximal ideals of $R^{*}$ and let $T^{*}=\left\{\left(\mathbf{m}_{i}, x-b_{i}\right) R^{*}\right\}_{i=1}^{t}$. Since $R^{*}$ is a finitely generated $R$-module, $R^{*}$ has only finitely many maximal ideals. By the Chinese Remainder Theorem there exist $c, d, e \in R^{*}$ so that $c \equiv b_{i}, d \equiv 0, e \equiv 1$ $\left(\bmod \mathbf{m}_{i}\right)$, for each $1 \leq i \leq t, d \neq 0$, and for any other maximal ideal $\mathbf{m}^{*}$ of $R^{*}$ we have $c \equiv 1, d \equiv 0, e \equiv 0\left(\bmod \mathrm{m}^{*}\right)$. Then for each positive integer $j,\left(e x-\left(c+d^{j}\right)\right) L[x] \cap R^{*}[x]$ is a height-one prime of $R^{*}[x]$ which is in the exactly-less-than set for $T^{*}$. This gives infinitely many height-one primes in the exactly-less-than set for $T^{*}$. Since only finitely many primes of the polynomial ring $L[x]$ have the same contraction in $K[x]$ and distinct primes of $K[x]$ have distinct contractions to $R[x]$, it follows that only finitely many primes in the exactly-less-than set for $T^{*}$ have the same contraction to $R[x]$. Since each prime ideal of $R^{*}[x]$ in the exactly-less-than set for $T^{*}$ contracts in $R[x]$ to a prime in the exactly-less-than set for $T$, we conclude that there are infinitely many height-one primes in the exactly-less-than set for $T$.

3.2 Remark. There is a natural extension of property $\left(\mathrm{P} 6_{n}\right)$ of Theorem 3.1 that applies in case $R$ is a one-dimensional semilocal domain with $m$ maximal ideals $\mathbf{m}_{1}, \ldots, \mathbf{m}_{m}$. If $\mathbf{m}_{i}$ is $n_{i}$-split for each $i$, where $n_{i}$ is a positive integer or $\infty$, it is easy to see via localization that if $T$ is a finite subset of $\mathscr{M}(\operatorname{Spec}(R[x]))$ such that $\left|T \cap \mathrm{G}\left(\mathbf{m}_{i}[x]\right)\right|>n_{i}$ for some $i$, then $\mathrm{L}_{\mathrm{e}}(T)=\varnothing$. On the other hand, if $\left|T \cap \mathrm{G}\left(\mathbf{m}_{i}[x]\right)\right| \leq n_{i}$, for all $i$, then an argument similar to that given in [HW, page 585] shows $\left|\mathrm{L}_{\mathrm{e}}(T)\right|=\infty$.

3.3 Theorem. (1) Let $m$ be a positive integer and let $R$ be a one-dimensional integral domain of valuative dimension one with exactly $m$ maximal ideals, $\mathbf{m}_{1}, \ldots, \mathbf{m}_{m}$. Then $\operatorname{Spec}(R[x])$ satisfies properties $(\mathrm{P} 1)-(\mathrm{P} 5)$ of Theorem 1.3 (1). 
(2) For each positive integer $n$, there exists a countable one-dimensional local domain $R$ such that $R$ is $n$-split with valuative dimension one; thus, $\operatorname{Spec}(R[x])$ satisfies the properties $(\mathrm{P} 0)-(\mathrm{P} 5)$ of Theorem $1.3(m=1)$, and $\left(\mathrm{P}_{n}\right)$ of Theorem 3.1.

(3) For each positive integer $m$, there exists a countable one-dimensional integral domain $R$ with exactly $m$ maximal ideals $\mathbf{m}_{1}, \ldots, \mathbf{m}_{m}$, and having the property that each $R_{\mathbf{m}_{i}}$ is 1-split and of valuative dimension one; thus, $\operatorname{Spec}(R[x])$ satisfies the properties (P0)-(P5) of Theorem 1.3, and each $\operatorname{Spec}\left(R_{\mathbf{m}_{i}}[x]\right)$ satisfies $\left(\mathbf{P} 6_{1}\right)$ of Theorem 3.1.

(4) More generally, for each ordered list of positive integers $m, n_{1}, \ldots, n_{m}$, there exists a countable one-dimensional integral domain $R$ with exactly $m$ maximal ideals $\mathbf{m}_{1}, \ldots, \mathbf{m}_{m}$, and having the property that $\boldsymbol{R}_{\mathbf{m}_{i}}$ is of valuative dimension one and is $n_{i}$-split for each $i, 1 \leq i \leq m$.

The picture of the prime spectrum of $R[x]$ from Theorem 3.3 is similar to the picture for Theorem 1.3; the information from $\left(\mathrm{P}_{n}\right)$ describes the inscrutable relations involving the rightmost box.

Proof. In part (1), (P1) is obvious. Property (P2) follows from [G, Theorem 30.9, page 360]. The proof of $(\mathrm{P} 3)$ is similar to that of $(\mathrm{P} 3)$ of Theorem 2.1.

Since $R$ has valuative dimension one, each $\mathbf{m}_{i}[x]$ is a height-one prime of $R[x]$. Also each $R[x] / \mathbf{m}_{i}[x]$ is a principal ideal domain with infinitely many maximal ideals, so each $\mathbf{m}_{i}[x]$ is a height-one nonmaximal $j$-element of $\operatorname{Spec}(R[x])$. Since each maximal ideal of $R[x]$ of height two contains one of the $\mathbf{m}_{i}[x]$, the $\mathbf{m}_{i}[x], 1 \leq i \leq m$, are precisely the height-one nonmaximal $j$-elements of $\operatorname{Spec}(R[x])$, whence we have (P4).

For (P5), note that if $P$ is a prime ideal of height one which is not maximal and not of the form $\mathbf{m}_{i}[x]$, then $\mathrm{G}(P)=\bigcup_{i=1}^{m}\left(\mathrm{G}\left(\mathbf{m}_{i}[x]\right) \cap \mathrm{G}(P)\right)$. This set is finite since $R[x]$ has Noetherian spectrum [OP].

To prove part (2), we use the example constructed in [HW] of an $n$-split, non-Noetherian domain. Let $k$ be a countable algebraically closed field, and let $V_{1}, \ldots, V_{n}$ be $n$ distinct rank-one valuation domains on $k(y)^{a}$, an algebraic closure of $k(y)$, such that $k$ is contained in each $V_{i}$. Then $V_{i}$ has the form $k+\mathscr{M}\left(V_{i}\right)$, where $\mathscr{M}\left(V_{i}\right)$ is the maximal ideal of $V_{i}$. By [N, (11.11)], $R^{\prime}=$ $V_{1} \cap \cdots \cap V_{n}$ is a one-dimensional domain with precisely $n$ maximal ideals $P_{i}=\mathscr{M}\left(V_{i}\right) \cap R^{\prime}$. Let $P=P_{1} \cap \cdots \cap P_{n}$, and let $R=k+P$, the set of all elements of $R^{\prime}$ of the form $a+p$, where $a \in k$ and $p \in P$. Then $R$ is an $n$-split one-dimensional local domain of valuative dimension one with integral closure $R^{\prime}$.

For part (3), the integral domain $R^{\prime}$ from the proof of part (2) above with $n=m$ has the desired properties since it is one-dimensional with precisely $m$ maximal ideals, the localization at each of which is a rank-one valuation domain and hence is of valuative dimension one. Moreover, $R^{\prime}$ is integrally closed with algebraically closed fraction field, so each of its prime ideals is 1-split.

To prove part (4), as in the proof of part (2), we take $n_{1}+\cdots+n_{m}$ distinct rank-one valuation domains on $k(y)^{a}$, each of which contains the field $k$. We partition these valuation domains into sets of $n_{1}, \ldots, n_{m}$ valuation domains, and for each $i, 1 \leq i \leq m$, we construct an $n_{i}$-split one-dimensional integral domain $R_{i}=k+P_{i}$, where $P_{i}$ is the intersection of the maximal ideals of the associated $n_{i}$ valuation domains. Using [H, (1.20)], we see that each $R_{i}$ is a localization of $R=\bigcap_{i=1}^{m} R_{i}$ and that each $\mathbf{m}_{i}=P_{i} \cap R$ is a maximal ideal of $R$. 
It follows that $R$ is a one-dimensional domain with precisely $m$ maximal ideals $\mathbf{m}_{1}, \ldots, \mathbf{m}_{m}$ where $R_{\mathbf{m}_{i}}=R_{i}$. This completes the proof of Theorem 3.3.

In connection with Theorem 3.3 we also have the following remarks, the proofs of which are straightforward.

3.4 Remarks. (1) If $U$ and $V$ are partially ordered sets satisfying the properties (P0)-(P5) of Theorem 1.3 for $m=1$ and $\left(\mathrm{P} 6_{n}\right)$ of Theorem 3.1, then $U \cong V$.

(2) If $R$ and $S$ are countable $n$-split one-dimensional local domains such that $R[x]$ and $S[x]$ are two-dimensional, then $\operatorname{Spec}(R[x]) \cong \operatorname{Spec}(S[x])$.

Using Theorems 3.1 and 3.3 along with [HW, Theorem 2.7], we have the following:

3.5 Corollary. If $R$ is a countable local 1-split (possibly non-Noetherian) onedimensional domain of valuative dimension one, then $\operatorname{Spec}(R[x])$ is orderisomorphic to $\operatorname{Spec}(H[x])$, where $H$ is the Henselization of $\mathbf{Z}_{(2)}$.

If $R$ is a countable $\infty$-split local (possibly non-Noetherian) one-dimensional domain of valuative dimension one, then $\operatorname{Spec}(R[x])$ is order-isomorphic to $\operatorname{Spec}\left(\mathbf{Z}_{(2)}[x]\right)$.

We now consider the case where $R$ is of valuative dimension greater than one.

3.6 Theorem. (1) If $R$ is an $n$-split one-dimensional local domain of valuative dimension greater than one, then $U=\operatorname{Spec}(R[x])$ satisfies the following properties:

(P1) $U$ has a unique minimal element $u_{0}$.

(P2) $U$ has dimension three.

(P3) $U$ has infinitely many height-one maximal elements.

(P4) $U$ has a unique height-two element, $u_{1}$. Furthermore, $\mathrm{G}\left(u_{1}\right)=\mathscr{M}(U)$ is infinite.

(P5) Regarding the height-one nonmaximal elements of $U$ :

(i) The set $S=\left\{\right.$ height-one nonmaximal elements $\left.u \mid u<u_{1}\right\}$ is infinite,

(ii) The set $S^{\prime}=\left\{\right.$ height-one nonmaximal elements $\left.u \mid u \nless u_{1}\right\}$ is infinite, and

(iii) for each $u \in S^{\prime}, \mathrm{G}(u)$ is finite.

(2) Let $n$ be a positive integer or $\infty$. There exists a countable one-dimensional local $n$-split integral domain $R$ of valuative dimension two. Thus $\operatorname{Spec}(R[x])$ satisfies the properties in (1) above and $\left(\mathrm{P} 6_{n}\right)$ of Theorem 3.1.

(3) For each positive integer $m$, there exists a countable one-dimensional integral domain $R$ with exactly $m$ maximal ideals $\mathbf{m}_{1}, \ldots, \mathbf{m}_{m}$, and having the property that each $R_{\mathbf{m}_{i}}$ is 1-split and of valuative dimension two.

(4) More generally, for each ordered list of positive integers $m, n_{1}, \ldots, n_{m}$, there exists a countable one-dimensional integral domain $R$ with exactly $m$ maximal ideals $\mathbf{m}_{1}, \ldots, \mathbf{m}_{n}$, and having the property that $R_{\mathbf{m}_{i}}$ is of valuative dimension two and is $n_{i}$-split for each $i, 1 \leq i \leq m$. 
Proof. For part (1), properties (P1) and (P3) follow as in Theorem 2.1, and (P2) holds by $[G,(30.9)]$. To see (P4), we again use that every prime ideal $P$ of $R[x]$, of height at least two, contains $\mathbf{m}$, and so $P \supseteq \mathbf{m}[x]$. Now $R[x] / \mathrm{m}[x]$ is obviously one-dimensional; since $R[x]$ is three-dimensional, $\mathbf{m}[x]$ must have height two and there can be no other height-two prime ideals of $R[x]$.

For (P5)(i) of Theorem 3.6, since $R$ has valuative dimension greater than one, there exists a valuation domain $V \supset R$ on the fraction field $K$ of $R$ which has two distinct primes $P_{1} \subset P_{2}$ with $R \cap P_{1}=R \cap P_{2}=\mathbf{m}$. We choose $\theta \in P_{2}-P_{1}$. Then in $R[\theta],(0) \subset \mathbf{m} R[\theta]=P_{1} \cap R[\theta] \subset P_{2} \cap R[\theta]=(\mathbf{m}, \theta) R[\theta]$; thus $R[\theta]$ has dimension two. Let $\theta=b / a$, where $a, b \in R$ and consider the kernel of the $R$-homomorphism $R[x] \rightarrow R[\theta]$ taking $x$ to $\theta$. This kernel is $(a x-b) K[x] \cap R[x]$, a height-one prime ideal of $R[x]$ which is properly contained in $\mathrm{m}[x]$. Indeed, for every positive integer $i, \theta^{i} \in P_{2}-P_{1}$, the $\theta^{i}$ are distinct elements of $K$, and the prime ideals $\left(a^{i} x-b^{i}\right) K[x] \cap R[x]$ are distinct and are properly contained in $\mathbf{m}[x]$. This gives infinitely many height-one elements $u<u_{1}$.

Since there exist infinitely many prime ideals of the form $(x-b) R[x]$, where $b \in R$, we have (P5)(ii); (P5)(iii) follows from the fact that $\operatorname{Spec}(R[x])$ is Noetherian.

To establish part (2), we start with a domain as in Example 1.5, that is, let $k$ be a countable algebraically closed field of characteristic zero, let $y, z$ be indeterminates over $k$, and let $R=k+z k(y)[z]_{(z)}$, with maximal ideal $\mathbf{m}=z k(y)[z]_{(z)}$. Then $R$ is a one-dimensional local integrally closed domain of valuative dimension two. Let $S$ be the integral closure of $R$ in an algebraic closure of the quotient field of $R$. To show that $R$ is $\infty$-split and thus that (2) holds in case $n=\infty$, we prove the following claim:

Claim 1. $S$ has infinitely many maximal ideals lying over $\mathbf{m}$.

Proof of Claim 1. For each positive integer $r$, the polynomial $x^{r}-z-1 \in R[x]$ is irreducible in $K[x]$, where $K$ is the fraction field of $R$, since it is linear in $z$, but it factors in $S[x]$ as a product of $r$ linear polynomials $x-a_{i}, 1 \leq i \leq r$. That is, $x^{r}-z-1=\prod_{i=1}^{r}\left(x-a_{i}\right)$, where $a_{i} \in S$. Now the ideals $\left(\mathbf{m}, x-a_{i}\right)$ are comaximal in $S[x]$, because the $a_{i}$ map to the $r$ distinct $r^{\text {th }}$ roots of unity in $R[x] / \mathrm{m}[x] \cong k[x]$. It follows that $S$ has at least $r$ distinct maximal ideals for each positive integer $r$. Therefore $S$ has infinitely many maximal ideals which proves Claim 1.

Now suppose $n<\infty$. Let $\mathscr{N}_{1}, \ldots, \mathscr{N}_{n}$ be $n$ distinct maximal ideals in $S$ and let $S_{i}$ be the localization of $S$ at $\mathscr{N}_{i}$, for $1 \leq i \leq n$. Then $S_{i}$ is a normal local domain of dimension one and valuative dimension two with residue field $k$ and fraction field $K^{a}$. Thus if $n=1$, any one of the $S_{i}$ gives the domain we want, since the $S_{i}$ are 1-split. We have $S_{i}=k+\mathscr{M}_{i}$, where $\mathscr{M}_{i}$ is the maximal ideal of $S_{i}$. Let $\mathscr{M}=\bigcap_{i=1}^{n} \mathscr{M}_{i}$ and $B=k+\mathscr{M}$. Then $B$ is a one-dimensional local domain having fraction field $K^{a}$ and valuative dimension two. Let $B^{\prime}$ be the integral closure of $B$ in $K^{a}$.

Claim 2. $B^{\prime}=\bigcap_{i=1}^{n} S_{i}$. 
Proof of Claim 2. Clearly $B \subseteq \bigcap_{i=1}^{n} S_{i}$, and $\bigcap_{i=1}^{n} S_{i}$ is integrally closed in $K^{a}$. Therefore $B^{\prime} \subseteq \bigcap_{i=1}^{n} S_{i}$. Suppose that $c \in \bigcap_{i=1}^{n} S_{i}$; for each $i, 1 \leq i \leq n$, write $c=a_{i}+d_{i}$, where $a_{i} \in k, d_{i} \in \mathscr{M}_{i}$. Consider $g(x)=\prod_{i=1}^{n}\left(x-a_{i}\right) \in B[x]$. Then

$$
c \in \bigcap_{i=1}^{n} S_{i} \Longrightarrow c-a_{j} \in S_{i}, \forall i, j \Longrightarrow c-a_{j} \in \bigcap_{i=1}^{n} S_{i}, \forall i, j .
$$

We have $g(c)=\prod_{i=1}^{n}\left(c-a_{i}\right)$ and $c-a_{i} \in \mathscr{M}_{i}$, so $g(c) \in \bigcap_{i=1}^{n} \mathscr{M}_{i}=\mathscr{M}$. Since $g(x)$ is monic, it follows that $c \in B^{\prime}$, proving Claim 2 .

Also $B^{\prime}$ has precisely $n$ distinct maximal ideals, $\mathscr{M}_{j} \cap B^{\prime}$, since each maximal ideal of $B^{\prime}$ is contained in some $\mathscr{M}_{j}$ and the $\mathscr{M}_{j} \cap S$ are distinct. Hence $B$ is local $n$-split of valuative dimension 2 .

For part (3), the integral domain $B^{\prime}=\bigcap_{i=1}^{n} S_{i}$ from the proof of part (2) above with $n=m$ has the desired properties. (As in 3.3 (4), it follows from [H, (1.20)] that the $S_{i}$ are precisely the localizations of $B^{\prime}$ at the maximal ideals of $B^{\prime}$.)

To prove part (4), we take $n_{1}+\cdots+n_{m}$ distinct maximal ideals of the integral domain $S$ constructed in the proof of part (2). The localizations of $S$ at these maximal ideals give us $n_{1}+\cdots+n_{m}$ one-dimensional normal local domains, each of valuative dimension two and each containing the field $k$ and having residue field isomorphic to $k$. We partition these one-dimensional local domains into sets of $n_{1}, \ldots, n_{m}$ local domains, and for each $i, 1 \leq i \leq m$, we construct an integral domain $R_{i}=k+P_{i}$, where $P_{i}$ is the intersection of the maximal ideals of the associated $n_{i}$ local domains. From part (2) it follows that $R_{i}$ is $n_{i}$-split and of valuative dimension two. Using [H, (1.20)], we see that $R=\bigcap_{i=1}^{m} R_{i}$ is a one-dimensional domain with precisely $m$ maximal ideals which can be listed $\mathbf{m}_{1}, \ldots, \mathbf{m}_{m}$ so that $R_{\mathbf{m}_{i}}=R_{i}$. Therefore $R$ has the stated properties of part (4). This completes the proof of Theorem 3.6.

Pictorially, a partially ordered set satisfying the properties in parts (1) and (2) of Theorem 3.6 looks like the diagram in Figure 2. The element $u_{1}$ is a sort of "waist" with an elbow out to one side. The interaction between the infinite sets at the top and those at the right is too complicated to display, but it is described by $\left(\mathrm{P} 6_{n}\right)$.

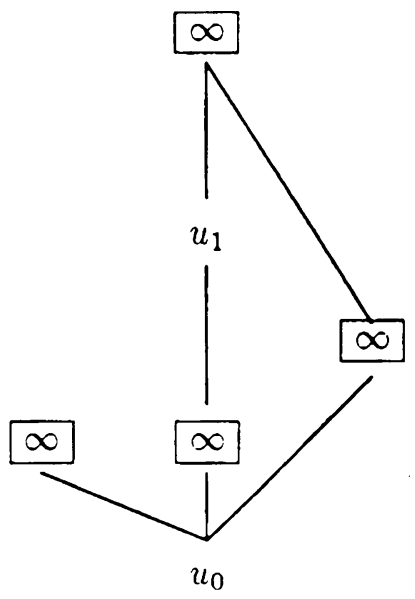

FIGURE 2 
In connection with Theorem 3.6 we also have the following remarks, the proofs of which are straightforward:

3.7 Remarks. (1) If $U$ and $V$ are countable partially ordered sets of dimension three satisfying the properties $(\mathrm{P} 1)-\left(\mathrm{P} 5_{2}\right)$ and $\left(\mathrm{P} 6_{n}\right)$ above, then $U \cong V$.

(2) If $R$ and $S$ are countable one-dimensional local $n$-split domains of valuative dimension greater than one, then $\operatorname{Spec}(R[x]) \cong \operatorname{Spec}(S[x])$.

3.8 Notes. (1) If $R$ is a one-dimensional domain with precisely $m$ maximal ideals and if the localization at each of these maximal ideals is of valuative dimension greater than one, then pictorially the partially ordered set $\operatorname{Spec}(R[x])$ is a generalization with exactly $m$ height-two "waists" of the picture given above for parts (1) and (2) of Theorem 3.6.

(2) Given any positive integers $r$ and $s$ and any lists $m_{1}, \ldots, m_{r}, n_{1}, \ldots$, $n_{s}$, where each $m_{i}$ and $n_{j}$ is either a positive integer or $\infty$, we believe it may be possible to construct a countable one-dimensional semilocal domain $R$ having precisely $r+s$ maximal ideals $\mathbf{m}_{1}, \ldots, \mathbf{m}_{r}, \mathbf{n}_{1}, \ldots, \mathbf{n}_{s}$ and where $R_{\mathbf{m}_{i}}$ is of valuative dimension one and $m_{i}$-split, while $R_{\mathbf{n}_{j}}$ is of valuative dimension greater than one and is $n_{j}$-split.

(3) If $P$ is a prime ideal of an integrally closed domain $R$, then as we mention in [HW, (1.7)], it seems plausible that $P$ can only be $1-, 2-$, or $\infty$ split.

\section{ACKNOWLEDGMENT}

The authors wish to thank the National Science Foundation for supporting this research. Wiegand thanks Purdue University for its hospitality during the academic year 1992/93, while this research was conducted. We thank the referee for a careful reading of the paper with helpful suggestions.

\section{REFERENCES}

[G] R. Gilmer, Multiplicative ideal theory, Marcel Dekker, New York, 1972.

[H] W. Heinzer, Noetherian intersections of integral domains II, Lecture Notes in Math., vol. 311, Springer-Verlag, 1973, pp. 107-119.

[HW] W. Heinzer and S. Wiegand, Prime ideals in two-dimensional polynomial rings, Proc. Amer. Math. Soc. 107 (1989), 577-586.

[HLW1] W. Heinzer, D. Lantz, and S. Wiegand, Prime ideals in birational extensions of polynomial rings, Commutative Algebra: Syzygies, Multiplicities and Birational Algebra (W. Heinzer, C. Huneke, and J. Sally, eds.), Contemp. Math., vol. 159, Amer. Math. Soc., Providence, RI, 1994, pp. 73-93.

[HLW2] __ Projective lines over one-dimensional semilocal domains and spectra of birational extensions, Algebraic Geometry and Applications (C. Bajaj, ed.), Collection of papers from Abhyankar's sixtieth birthday conference, Springer-Verlag, New York (to appear).

[J] P. Jaffard, Théorie de la dimension dans les anneaux de polynomes, Mémorial des Sciences Mathématiques, Gauthier-Villars, Paris, 1960.

[M] H. Matsumura, Commutative ring theory, Cambridge Stud. Adv. Math., vol. 8, Cambridge Univ. Press, Cambridge, 1989.

[Mc] S. McAdam, Intersections of height two primes, J. Algebra 49 (1977), 315-321.

[N] M. Nagata, Local Rings, Interscience, New York, 1962.

[OP] J. Ohm and Pendleton, Rings with Noetherian spectrum, Duke Math. J. 35 (1968), 631-640. 
[S] A. Seidenberg, A note on the dimension theory of rings, Pacific J. Math. 3 (1953), 505-512.

[rW1] R. Wiegand, Homeomorphisms of affine surfaces over a finite field, J. London Math. Soc. (2) 18 (1978), 28-32.

[rW2] _ The prime spectrum of a two-dimensional affine domain, J. Pure Appl. Algebra 40 (1986), 209-214.

Department of Mathematics, Purdue University, West Lafayette, Indiana 47907-1395

Department of Mathematics, University of Nebraska, Lincoln, Nebraska 68588-0323 